MATHEMATICS OF COMPUTATION

Volume 73 , Number 246 , Pages 653-657

S $0025-5718(03) 01591-6$

Article electronically published on October 17, 2003

\title{
GAUSSIAN ELIMINATION IS STABLE FOR THE INVERSE OF A DIAGONALLY DOMINANT MATRIX
}

\author{
ALAN GEORGE AND KHAKIM D. IKRAMOV
}

\begin{abstract}
Let $B \in M_{n}(\mathbf{C})$ be a row diagonally dominant matrix, i.e.,

$$
\sigma_{i}\left|b_{i i}\right|=\sum_{\substack{j=1 \\ j \neq i}}^{n}\left|b_{i j}\right|, \quad i=1, \ldots, n,
$$

where $0 \leq \sigma_{i}<1, i=1, \ldots, n$, with $\sigma=\max _{1 \leq i \leq n} \sigma_{i}$. We show that no pivoting is necessary when Gaussian elimination is applied to $A=B^{-1}$. Moreover, the growth factor for $A$ does not exceed $1+\sigma$. The same results are true with row diagonal dominance being replaced by column diagonal dominance.
\end{abstract}

\section{INTRODUCTION}

We begin with a quotation from N. Higham's paper [1]: "There are three main classes of matrix for which it is known to be safe not to pivot when computing an LU factorization: matrices diagonally dominant by rows or columns, Hermitian positive definite matrices, and totally nonnegative matrices." Then the author proceeds to "identify another class of matrices with this highly desirable property: complex symmetric matrices whose real and imaginary parts are both positive definite." In this short article we extend the set of matrices having this property to include matrices whose inverses are matrices diagonally dominant by rows or columns, and we show that the growth factor for such matrices is bounded by two. The reader will find the proof in Section 3, with preliminary material required for the proof contained in Section 2.

\section{Preliminary FACts}

Let $A \in M_{n}(\mathbf{C})$, the set of $n \times n$ complex matrices. For an index set $\alpha \subseteq$ $\{1, \ldots, n\}$, we denote the principal submatrix of $A$ that lies in the rows and columns indexed by $\alpha$ as $A(\alpha)$ and its complementary principal submatrix as $A\left(\alpha^{\prime}\right)$. The following lemma is of crucial importance in Section 3.

Received by the editor February 13, 2002 and, in revised form, August 1, 2002.

2000 Mathematics Subject Classification. Primary 65F10.

Key words and phrases. Gaussian elimination, growth factor, diagonally dominant matrices, Schur complement.

This work was supported by Natural Sciences and Engineering Research Council of Canada grant OGP0008111. 
Lemma 1. Let $A \in M_{n}(\mathbf{C})$ be a nonsingular matrix, and $B=A^{-1}$. Let $\alpha$ be a subset of $\{1, \ldots, n\}$. The inequality

$$
|\operatorname{det} A| \leq \rho|\operatorname{det} A(\alpha)|\left|\operatorname{det} A\left(\alpha^{\prime}\right)\right|
$$

with a positive scalar $\rho$ holds if and only if the similar inequality

$$
|\operatorname{det} B| \leq \rho|\operatorname{det} B(\alpha)|\left|\operatorname{det} B\left(\alpha^{\prime}\right)\right|
$$

holds for the matrix $B$.

Proof. Inequality (2) is just another form of (1). This can be seen from the relations

$$
\begin{aligned}
\operatorname{det} B & =\frac{1}{\operatorname{det} A}, \\
\operatorname{det} B(\alpha) & =\frac{\operatorname{det} A\left(\alpha^{\prime}\right)}{\operatorname{det} A}, \\
\operatorname{det} B\left(\alpha^{\prime}\right) & =\frac{\operatorname{det} A(\alpha)}{\operatorname{det} A} .
\end{aligned}
$$

The last two equalities are particular cases of a general formula that connects minors in $B$ and $A$ (see formula (33) in [2, Chapter1]).

We say that $B \in M_{n}(\mathbf{C})$ is a (row) diagonally dominant matrix (d.d. matrix, for short) if

$$
\sigma_{i}\left|b_{i i}\right|=\sum_{\substack{j=1 \\ j \neq i}}^{n}\left|b_{i j}\right|, \quad i=1, \ldots, n,
$$

where $0 \leq \sigma_{i}<1, i=1, \ldots, n$. The quantity

$$
\sigma=\max _{1 \leq i \leq n} \sigma_{i}
$$

will be called the dominance factor of $B$.

Lemma 2. Let $B$ be a d.d. matrix, and let $B^{-1}=A=\left(a_{i j}\right)$. Then, for $i=1, \ldots, n$,

$$
|\operatorname{det} B| \leq\left(1+\sigma_{i}\right)\left|b_{i i}\right|\left|B_{i}\right|,
$$

where $B_{i}$ is the cofactor of $b_{i i}$, and

$$
\left|a_{j i}\right| \leq \sigma_{j}\left|a_{i i}\right|, \quad j \neq i .
$$

Both assertions of the lemma can be found in [3, Sections 4, 6, and 7]. Inequality (6) says that, in each column of the inverse matrix $A$, the element with the largest modulus is on the main diagonal.

Suppose that a nonsingular $n$-by- $n$ matrix $A$ with nonvanishing leading principal minors undergoes Gaussian elimination with no pivoting. After $k$ steps of the elimination have been completed, we have an order $n-k$ matrix that has yet to be processed. This matrix is alternatively called the active submatrix (after the $k$ th step) or the Schur complement. In the latter case, it is denoted as $A / A(\alpha)$, where

$$
\alpha=\{1, \ldots, k\} .
$$

Lemma 3. It holds that

$$
(A / A(\alpha))^{-1}=B\left(\alpha^{\prime}\right),
$$

where $B=A^{-1}$. 
This is a well-known relation (see, for example, [4. Sec. 0.7.3]).

Let $a_{i j}^{(k)}(i, j=k+1, \ldots, n)$ be the entries of the Schur complement $A / A(\alpha), \alpha$ being the index set in (7). The quantity

$$
\rho_{n}(A)=\frac{\max _{i, j, k}\left|a_{i j}^{(k)}\right|}{\max _{i, j}\left|a_{i j}\right|}
$$

is called the growth factor for $A$.

The properties of d.d. matrices related to Gaussian elimination are widely known. We state those we need in Section 3 in the lemma below.

Lemma 4. Let $B \in M_{n}(\mathbf{C})$ be a d.d. matrix with the row dominance factors $\sigma_{i}$ (see (3)). Then:

(1) Gaussian elimination is applicable to $B$ under any diagonal pivoting order.

(2) The diagonal dominance property is inherited by active submatrices. In other words, each Schur complement $B / B(\alpha)$ is also a d.d. matrix. Moreover, for each $i$, the row dominance factor $\sigma_{i}^{\prime}$ for $B / B(\alpha)$ does not exceed the corresponding factor $\sigma_{i}$ for $B$ (assuming that the original row indices of $B$ remain "attached" to the rows in $B / B(\alpha)$ ).

\section{THE MAIN RESUlT}

We now prove

Theorem 1. Let $A \in M_{n}(\mathbf{C})$ be a nonsingular matrix such that $B=A^{-1}$ is a d.d. matrix with the dominance factor $\sigma$ (see (4)). Then

$$
\rho_{n}(A) \leq 1+\sigma .
$$

Proof. By Lemma 2, $a_{11}$ is the entry with the largest modulus in the first column. Thus, $a_{11}$ can be taken as the pivot for the first step of elimination. Setting $\alpha=\{1\}$, we see from (8) that $A / A(\alpha)$ has the d.d. matrix $B\left(\alpha^{\prime}\right)$ as its inverse. Hence, $a_{22}^{(1)}$ is the entry with the largest modulus in the first column of $A / A(\alpha)$ and can be taken as the pivot for the second step. Continuing in this way, we conclude that no permutations are needed to perform Gaussian elimination (GE) for A. Moreover, GE with no pivoting as applied to $A$ is the same as GE with partial pivoting.

In fact, relation (6) means that the entry with the largest modulus in the entire matrix $A$ belongs to the main diagonal. The same is true for all Schur complements $A / A(\alpha)$. Hence, to bound $\rho_{n}(A)$, we have to examine only the behavior of the diagonal entries in the course of elimination. Assume that

$$
M=\max _{r, s, t}\left|a_{r s}^{(t)}\right|
$$

is attained when $r=s=i, t=k$. Define

$$
\alpha=\{1, \ldots, k\}, \quad \beta=\alpha \cup\{i\} .
$$

Then

$$
M=\frac{|\operatorname{det} A(\beta)|}{|\operatorname{det} A(\alpha)|} .
$$

The inverse of $A(\beta)$ is $B / B\left(\beta^{\prime}\right)$. According to Lemma $4, B / B\left(\beta^{\prime}\right)$ is a d.d. matrix, and its row dominance factors $\sigma_{i}^{\prime}$ do not exceed the corresponding factors $\sigma_{i}$ for $B$. 
It follows that an inequality of type (5) is valid for $B / B\left(\beta^{\prime}\right)$ and then, by Lemma 1 , the similar inequality

$$
|\operatorname{det} A(\beta)| \leq\left(1+\sigma_{i}\right)\left|a_{i i}\right||\operatorname{det} A(\alpha)|
$$

holds for $A(\beta)$. Relations (11) and (12) imply that

$$
\rho_{n}(A)=\frac{M}{\max _{r}\left|a_{r r}\right|} \leq\left(1+\sigma_{i}\right) \frac{\left|a_{i i}\right|}{\max _{r}\left|a_{r r}\right|} \leq 1+\sigma .
$$

The theorem is proved.

Remark. It can be shown that bound (10) is, in fact, the strict inequality $\rho_{n}(A)<$ $1+\sigma$, when $0<\sigma<1$. Note that $1+\sigma$ is also a bound for the growth factor of the d.d. matrix $B=A^{-1}$.

Remark. It is clear that, in the argument above, the row diagonal dominance could be replaced by the column diagonal dominance. Thus, we have

Theorem 2. Let $A \in M_{n}(\mathbf{C})$ be a nonsingular matrix such that $B=A^{-1}$ is a matrix diagonally dominant by columns with the (column) dominance factor $\sigma$. Then

$$
\rho_{n}(A) \leq 1+\sigma .
$$

The results of this paper can be extended to block matrices with the block diagonal dominance property (see 9 Chapter 12]). This will be the subject of our forthcoming paper.

\section{Acknowledments}

We wish to thank the referee for the very careful reading of the paper and many helpful remarks. In particular, the referee has pointed out paper [5], where bounds were obtained on the growth factor for inverses of $H$-matrices. The latter class contains the matrices we consider in our paper. However, for these matrices, the bounds in [5] are different from our bounds (10) and (13). In general, they are weaker than ours with the notable exception of the case when $A^{-1}$ is an $M$-matrix. In this case, the authors of [5] were able to show that $\rho_{n}(A)=1$.

Following [5], we list some applications where systems of linear equations $A x=b$ with $A$ being the inverse of an $M$-matrix are encountered. These are the solution of certain integral equations [6], a time series approach to numerical differentiation [7], and certain physical problems involving coupled oscillators [8].

\section{REFERENCES}

1. N.J. Higham, Factorizing complex symmetric matrices with positive definite real and imaginary parts, Math. Comp. 67 (1998), 1591-1599. MR 99a:65049

2. F.R. Gantmacher, The Theory of Matrices, Chelsea, New York, 1959. MR 21:6372c

3. A.M. Ostrowski, Note on bounds for determinants with dominant principal diagonal, Proc. Amer. Math. Soc. 3 (1952), 26-30. MR 14:611c

4. R.A. Horn and C.R. Johnson, Matrix Analysis, Cambridge University Press, 1985. MR 87e:15001

5. M. Neumann and R.J. Plemmons, Backward error analysis for linear systems associated with inverses of H-matrices, BIT 24 (1984), 102-112. MR 85f:65027

6. R.A. Willoughby, The inverse M-matrix problem, Linear Algebra Appl. 18 (1977), 75-94. MR 57:12561

7. R. Andersen and R. Bloomfield, A time series approach to numerical differentiation, Technometrics 16 (1974), 69-75. MR 53:1889 
8. H.S. Leff, Correlation inequalities for coupled oscillators, J. Math. Phys. 12 (1971), 569-578. MR 43:8322

9. N.J. Higham, Accuracy and Stability of Numerical Algorithms, SIAM, Philadelphia, 1996. MR 97a:65047

School of Computer Science, University of Waterloo, Waterloo, Ontario, Canada

Faculty of Computational Mathematics and Cybernetics, Moscow State University, 119992 Moscow, Russia

E-mail address: ikramov@cs.msu.su 